\title{
Single, binary and successive patterning of charged nanoparticles by electrophoretic deposition
}

\author{
Eliza Sopubekova • Güneş Kibar • \\ E. Yegan Erdem (1)
}

Received: 2 May 2021 / Accepted: 10 November 2021 / Published online: 19 November 2021

(C) The Author(s), under exclusive licence to Springer Nature B.V. 2021

\begin{abstract}
Deposition of nanoparticles on a substrate in a controlled manner leads to the formation of multifunctional surfaces and therefore devices. Electrostatic forces can be utilized to manipulate different types of materials such as magnetic, insulating, conducting, semiconducting, organic and inorganic, without altering the chemistry of the surface. However, simultaneous and successive electrophoretic deposition (EPD) methods are not fully utilized for nanoparticles with different characteristics. In this work, electrostatic forces are applied to direct and position charged nanoparticles suspended in aqueous dispersions on desired areas of the surface. Assemblies of particles are obtained by electrostatic attraction generated by gold electrodes of sizes from $500 \mathrm{~nm}$ to $50 \mu \mathrm{m}$ that are fabricated by thermal evaporation. Different types of charged nanoparticles
\end{abstract}

Supplementary Information The online version contains supplementary material available at https://doi. org/10.1007/s11051-021-05368-1.

E. Sopubekova · E. Y. Erdem

National Nanotechnology Research Center (UNAM), Ankara, Turkey

G. Kibar

Department of Materials Engineering, Adana Alparslan Türkeş University, Adana, Turkey

E. Y. Erdem $(\bowtie)$

Department of Mechanical Engineering, İ. D. Bilkent

University, Ankara, Turkey

e-mail: yeganerdem@bilkent.edu.tr were simultaneously attracted towards different locations of the surface by means of EPD; as a result, alternating nanoparticle patterns and particle deposition on the same designated areas for forming composite areas are obtained. Assemblies formed from positively charged silver nanoparticles and negatively charged fluorescent latex and silica nanoparticles are demonstrated. The position of metallic-, polymericand inorganic-based nanoparticles is controlled by the design of electrode geometry.

Keywords Nanoimprinting $\cdot$ Nanoparticle assembly $\cdot$ Electrophoretic deposition .

Nanofabrication

\section{Introduction}

Today, there have been remarkable advancements in the fabrication, processing and application of materials within the nanometre size range. Nanoparticles attracted extensive attention due to their notable differences in physical, optical and electronic properties compared to bulk materials. These properties are dictated by particle size and shape, and they offer possibilities in optical, electronic, sensing and biomedical applications. For instance, semiconductor nanoparticles known as quantum dots (QDs) have only a few nanometres of radius, and their size is on the same order as the exciton Bohr radius. Thus, by adjusting the size of a crystal, absorption 
and emission spectra can be controlled. Besides, the relatively sharp absorption and emission features incorporate a wide spectral range from ultraviolet to infrared (Murray et al. 1993). These aspects of QDs make them suitable candidates for various applications. Some of these applications are optoelectronic devices such as imaging of biological systems (Ding et al. 2019), LEDs (Liu et al. 2020), solar cells (Selopal et al. 2017; Beattie et al. 2017) and photodetectors (Shen et al. 2019). Metallic nanoparticles such as gold ( $\mathrm{Au}$ ) and silver $(\mathrm{Ag})$ are biocompatible, and due to their particle-related photonic, electronic and plasmonic characteristics, they can be used in biosensing (Song et al. 2014), cancer diagnosis and treatment (Park et al. 2018), and medical imaging (Banstola et al. 2018). Gold nanoparticle aggregates were also studied for their photoluminescence dependence where their distribution on a surface was an important parameter (Abdellatif et al. 2016; Intartaglia et al. 2016). Other types of materials such as magnetic and polymer nanoparticles have also been studied for potential applications in biomedicine (Pankhurst et al. 2016; Hunter et al. 2012). The combination of different types of nanomaterials enhances the functionality and efficiency of the devices due to their collective features. For instance, energy sources such as solar cells, lithium-ion batteries and fuel cells can be manufactured by placing different types of semiconductor particles on the same surface (Goesmann and Feldmann 2010). Furthermore, it is necessary to place or 'print' the particles on a surface in a controlled manner, since it is a crucial factor affecting the device performance and functionality (Terris and Thomson 2005).

Various methods are established in order to print nanoparticles on a surface. One of these methods is called a template-assisted assembly, also known as nanoimprint lithography (Park et al. 2008; Demko et al. 2011; Jeong et al. 2012; Jiang et al. 2016; Zhang et al. 2018a). It uses a flexible polymer template to obtain the desired arrangement of nanoparticles on the surface. The drawback of this method is that the residual layer, which must be etched away, is partially left on the substrate after the pattern transfer, and it is limited for the patterning of only one type of nanoparticle on the surface. Another prominent process known for controlled placement of particles is inkjet printing (Kamyshny and Magdassi 2014; An et al. 2015). The particles that are suspended in a fluid are ejected to the surface through the nozzle to create a pattern. This method is popular for being simple and additive, and the particle solution is not wasted because etching of the residual layer is not necessary. Nonetheless, the resolution of the printed pattern is around 20-50 $\mu \mathrm{m}$ (An et al. 2015), and the geometry control over the patterns is bounded. For obtaining finer features, methods with higher resolutions should be preferred.

Substitutions for the template-based methods are the ones that employ electrostatic forces to manipulate and direct the nanoparticles to form patterns. There are two approaches that utilize these forces. The first approach is directing neutral nanoparticles in a fluid by means of dielectrophoretic forces in a non-uniform electric field (Wood et al. 2013). The second approach is to initiate the diffusion of charged nanoparticles towards the oppositely charged substrate surface by using the Coulomb forces also known as electrophoretic forces (Jacobs et al. 2002; Barry et al. 2003, 2005). Over the last few years, the most versatile alternative for the controlled assembly of charged nanoparticles is believed to be the atomic force microscope (AFM) nanoxerography (Palleau et al. 2010, 2011; Moutet et al. 2015; Morales et al. 2018). Since the cantilever tip enables writing both positive and negative charges on the surface, it was possible to create the assemblies from two different types of particles (Palleau et al. 2011; Morales et al. 2018). However, the size of a single scan area is limited $(150 \mu \mathrm{m} \times 150 \mu \mathrm{m})$, and the required voltages for sufficient polarization are high $(\sim 80 \mathrm{~V})$.

The electrophoretic deposition (EPD) technique is utilized in this work because of its simplicity, applicability to all types of nanomaterials that have a net surface charge and possibility of controlling the shape of patterns. In literature, EPD is commonly used to produce thin films and composites of nanoparticles and biomaterials adsorbed on continuous metallic surfaces (Sarkar and Nicholson 1996; Guo and Liu 2012; Seuss and Boccaccini 2013; Mills et al. 2020). Only a few studies on shape-controlled coatings were reported. One of them was proposed by Majetich et al. (Oberdick and Majetich 2013), where they located the iron oxide nanoparticles to the desired areas by putting the geometric barriers made of hydrogen silsesquioxane (HSQ) on the electrode surface. Other recent studies also showed that controlled gold nanoparticles (Zhang et al. 2018b) and polystyrene bead 
(Qian et al. 2015) arrays can be assembled on a conductive substrate by using PMMA templates. These methods were limited in patterning a single type of nanoparticle as charging of only selected areas on the surface was not possible. Few studies were reported on the patterning of biomolecules by using EPD (Chavez-Valdez et al. 2013; Sikkema et al. 2020). Binary patterns were obtained by Gao et al., where two types of CdTe nanocrystals were positioned next to each other on a structured ITO electrode (Gao et al. 2002). However, this method requires post-processing (removal of the complementary resist after the patterning) and creates difficulties at higher resolutions and with complex geometries since the polarity of each electrode stripe has to be controlled separately for every deposition.

This study addresses the challenge of the single and binary assembly of nanoparticles on structured electrodes by using EPD. The method does not require any template, mask or post-processing, where the geometry and the resolution of patterns are controlled by the shape and sizes of the electrodes. An alternative pattern formation and composite surface formation by using two different types of nanoparticles are demonstrated.

\section{Experimental methods}

\section{Materials}

Silver nitrate (AgNO3) salt (209,139, Germany) and a stabilizer polyethyleneimine (PEI) branched average Mw 25,000 (408,727, Germany) were purchased from Sigma-Aldrich. Distilled deionized (DDI) water was obtained from Millipore/Direct Q-3UV water purification system located in our clean room at National Nanotechnology Research Center in Ankara, Turkey. Fluorescent latex (MFCD00131492, Germany) nanoparticles were purchased from Sigma-Aldrich, and silica nanoparticles (42-01-301, Germany) were purchased from Micromod Partikel Technologie $\mathrm{GmbH}$.

Nanoparticle synthesis and characterization

Three different nanoparticles were chosen to show the versatility of the technique: metallic (silver), polymeric (polystyrene latex) and silica nanoparticles. These are widely used in bioimaging (Yoo et al. 2018), sensing (Peng et al. 2018) and optoelectronic devices (Belusso et al. 2019). These particles are all spherical and in aqueous solutions and have either a positive or negative surface charge due to their capping agents. Only silver particles were synthesized in our laboratory following the method described by Sharonova et al. (Sharonova et al. 2016); others were purchased. In the synthesis of silver nanoparticles, the reaction medium was prepared by dissolving $2.5 \%$ (wt) PEI in 10-ml DDI water and was magnetically stirred at $250 \mathrm{rpm}$ and heated to $90{ }^{\circ} \mathrm{C}$ in an oil bath. The silver precursor was prepared by dissolving $0.125 \mathrm{~g} \mathrm{AgNO} 3$ in 250- $\mu$ LDI. This precursor solution was added to the reaction medium and was mixed at $90{ }^{\circ} \mathrm{C}$ for $1 \mathrm{~h}$. The colour of the dispersion medium was changed from yellowish to metallic black or dark grey colour. The positively charged Ag-NPs were collected from the medium after centrifugation at 15,000 rpm for $15 \mathrm{~min}$. The black precipitate was washed with DDI water. The positively charged AgNPs were kept at room temperature in DDI water or absolute ethanol for further studies. All nanoparticle dispersions showed high stability and monodispersity. Zeta $(\zeta)$ potentials and the size of the nanoparticles were measured in an aqueous medium by using Zeta Sizer (Malvern Zetasizer Lab, Netherlands). The obtained data are given in the supplementary information as Figure S1. The physical properties of nanoparticles used in the experiments are given in Table 1.

In addition to the properties listed in Table 1, latex particles are fluorescent green $(470 / 505 \mathrm{~nm})$ and silica particles are fluorescent red $(569 / 585 \mathrm{~nm})$, which

Table 1 Properties of nanoparticles used in this study

\begin{tabular}{llll}
\hline Nanoparticle & Diameter $(\mathrm{nm})$ & $\zeta$ potential $(\mathrm{mV})$ & Capping agent \\
\hline Silver & $80-100$ & $+61.8 \pm 11$ & Polyethyleneimine (PEI) \\
Latex & 30 & $-71.8 \pm 9$ & Carboxylate $(-\mathrm{COOH})$ \\
Silica & 30 & $-24.8 \pm 9$ & Amine $\left(-\mathrm{NH}_{2}\right)$ \\
\hline
\end{tabular}


makes them available to spot under the fluorescent microscope (DIC Equipped Inverted, ZEISS Observer A1, Germany).

\section{Fabrication of electrodes}

Electrodes used in this work were fabricated in both micrometre and submicrometre sizes in the clean room of the National Nanotechnology Research Center (UNAM). The electrodes in the micrometre size range were fabricated by the conventional etch-back technique. In this process, 4-inch silicon wafers with a 90-nm thick silicon dioxide $\left(\mathrm{SiO}_{2}\right)$ were coated with a 5 -nm layer of chromium followed by a 100-nm gold layer by thermal evaporation (Vaksis, Midas 2M3T1ICP,
Turkey). Next, the positive photoresist was spin coated and exposed to UV light (Karlsüss mjb3, Germany). The desired electrode patterns were obtained after wet etching of gold and chromium. The schematics of the fabrication steps are shown in Figure S2 (please see the supplementary information). The electrodes in submicrometre sizes were obtained by utilizing the electron beam lithography (EBL) (FEI NNS 600, Nova, Israel) tool instead of regular UV lithography. In this case, electrode patterns were formed by the electron beam on silicon substrates coated with PMMA resist followed by the coating of $5 \mathrm{~nm}$ of $\mathrm{Cr}$ and $100 \mathrm{~nm}$ of Au. Later substrates were left in the acetone for $8 \mathrm{~h}$ for lift-off. The electrodes are successfully fabricated and shown in Figs. 1 and 2.
Fig. 1 SEM images of gold electrodes fabricated with UV photolithography and lift-off. a Parallel electrodes. b Tori- shaped electrodes

Fig. 2 a-c SEM images of 500-nm comb-like electrode arrays that were fabricated with EBL. Images are taken at different scales
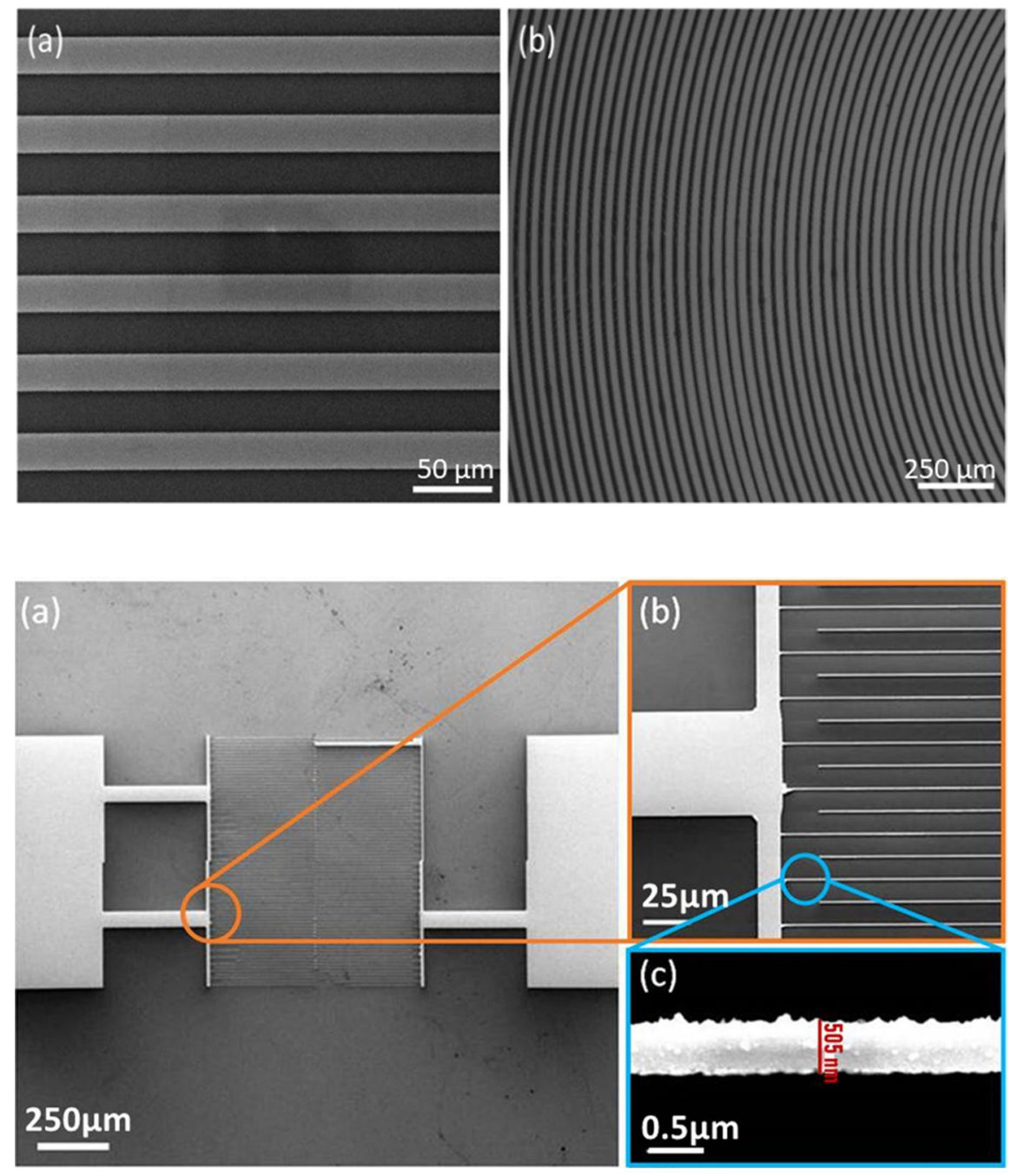
Experimental procedure

The experimental set-up is composed of a patterned electrode, a counter electrode and a power source. Two electrodes facing each other are vertically immersed into the nanoparticle solution, and voltage is applied across them. The electrophoretic cell containing silica nanoparticle solution is shown in Figure S3. Instead of a DC power supply, signal generator, amplifier and oscilloscope are used to generate the pulses of voltage. Using pulsed DC voltage instead of continuous voltage suppresses the bubble formation in an aqueous solution and allows nanoparticle diffusion without any disturbance (Besra et al. 2009). The electric field is generated in the solution between these electrodes, and nanoparticles are driven towards the oppositely charged electrode. Electrolysis at the cathode and anode disrupts the particle assembly in an aqueous solution due to the formation of bubbles; therefore, pulsed DC voltage was applied instead of continuous (Besra et al. 2009). Pulsed DC voltage was generated by a function generator (GWInstek SFG-2004, Taiwan) and monitored with an oscilloscope (Keysight, InfiniVision DSO-X 2012A, USA). Pulse width lower than $1 \mathrm{~ms}$ was applied at a $50 \%$ duty cycle; there was a very little amount of bubble formation on the electrodes. The reason is that the electrolysis is significantly decreased by shocking the reaction system with constantly pulsing current voltage. Namely, $\mathrm{H}_{2}$ is produced from proton reduction, and $\mathrm{O}_{2}$ is produced from hydroxyl in water electrolysis. The frequency of the pulsed voltage is high enough to slow down this reaction. The signal was enhanced with the help of an amplifier. The experimental set-up and procedure are schematically shown in Fig. 3.
The EDP kinetic model that describes the amount of particles deposited on the electrode surface was proposed by Hamaker (Hamaker 1940). According to this model, the deposited mass per unit area is dependent on the suspension concentration, particle properties such as electrophoretic mobility, electric field strength, deposition area and deposition time.

\section{Results and discussion}

Singular patterning of nanoparticles

Assembling a single type of nanoparticles on a surface by electrostatic forces was studied with silver, latex and silica nanoparticles to show the versatility of this method in patterning both metal and nonmetal (organic and inorganic) nanoparticles.

Initially, gold electrodes of width $20 \mu \mathrm{m}$ were used to assemble positively charged silver nanoparticles. Negatively biased $20 \mathrm{~V}$ was applied across the torishaped electrodes for $5 \mathrm{~min}$, while they were exposed to the aqueous silver nanoparticle solution with a concentration of $0.12 \mathrm{mg} / \mathrm{ml}$. The deposition efficiency of silver nanoparticles on the gold electrode surface was calculated as $90 \%$ by counting the particles on electrodes with the image processing program ImageJ (NIH, USA).

In electrophoretic deposition, the amount of the patterned nanoparticles on the surface increases with the deposition time until saturation is reached. In silver nanoparticle patterning, saturation was obtained within $10 \mathrm{~min}$; after which, there was not any noticeable change in the assembled number of particles. On the other hand, at voltages lower than $20 \mathrm{~V}$, less amount of deposited $\mathrm{Ag}$ nanoparticles was observed.

Fig. 3 Schematic of the experimental apparatus and nanoparticle assembly on the patterned electrode
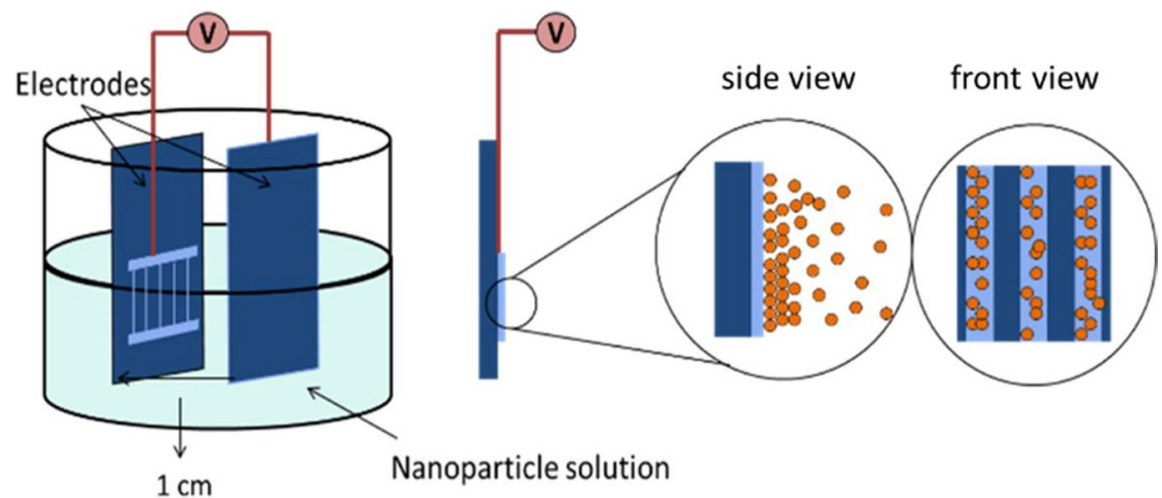

$1 \mathrm{~cm}$

Nanoparticle solution 
Scanning electron microscopy (SEM) (QUANTA 200EF, FEI, USA) images of patterned electrodes are shown in Fig. 4.

In the experiments with latex nanoparticles, $15 \mathrm{~V}$ of potential for 5 min was sufficient for the diffusion and assembly. The decrease in the required voltage and time is mainly due to their significantly high $\zeta$ potential $(\sim-70 \mathrm{mV})$. Latex nanoparticles illuminate fluorescent green colour, and this allows them to be differentiated easily under the fluorescent microscope.
Fluorescent micrographs of patterns of latex nanoparticles on electrode surfaces are shown in Figs. 5 and 6 . Besides a few aggregations on some areas, the patterning was successful.

Patterning of latex nanoparticles was also successful on submicrometre-sized electrode surfaces where the surface area of the electrodes fabricated with EBL is considerably smaller than the surface area of the electrodes obtained by photolithography $\left(800 \mu \mathrm{m}^{2}\right.$ vs. $8 \mathrm{~mm}$ for comb-like line electrode geometries). This
Fig. 4 a, b Representative SEM images of silver nanoparticles patterned on the tori-shaped electrodes at two different scales

Fig. 5 a, b Fluorescent micrographs of latex nanoparticle assemblies on the tori-like electrode surface at two different scales
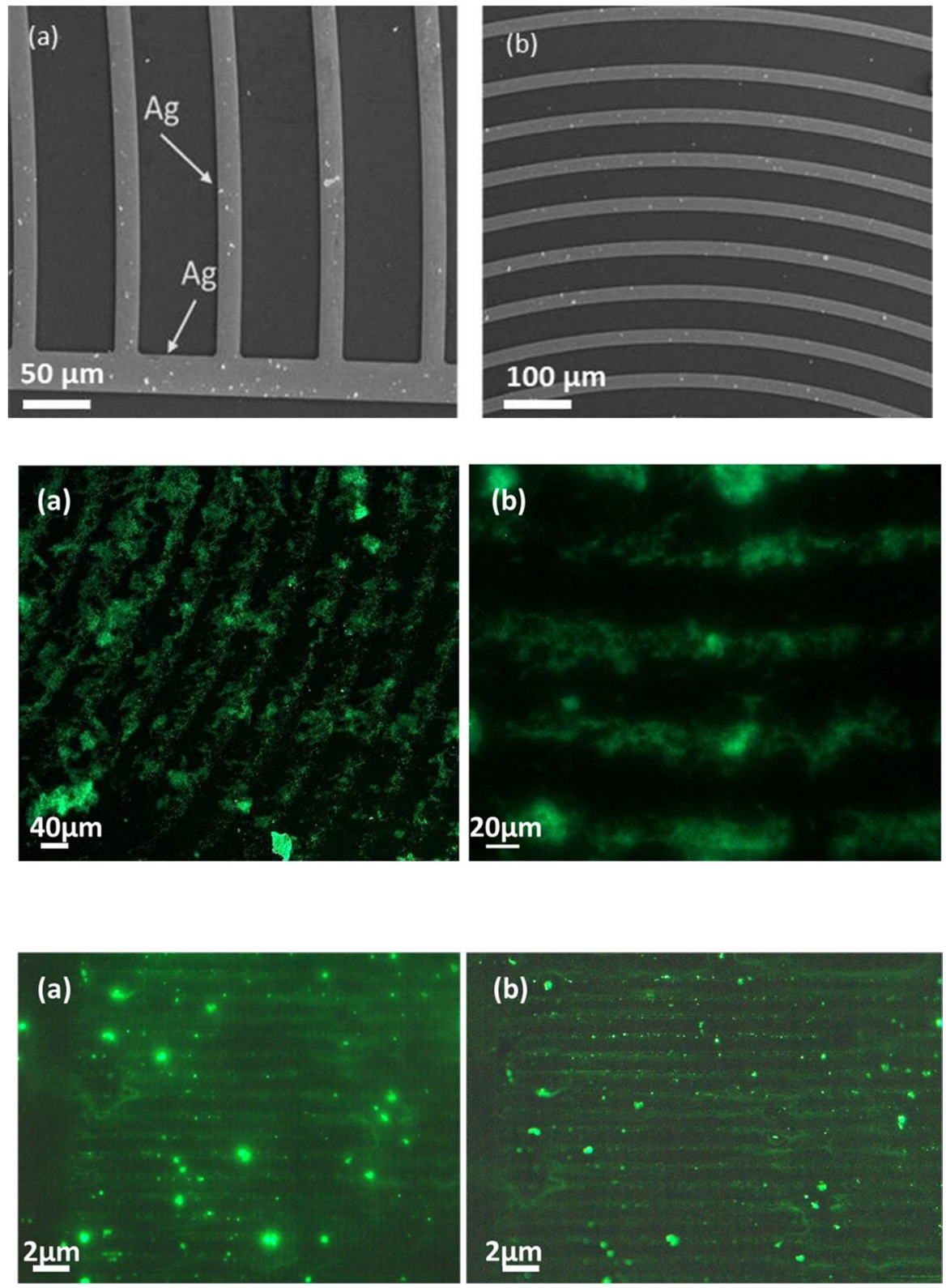

Fig. 6 a, b Latex nanoparticle patterns on 500-nm wide electrodes 
directly affects the electric field strength generated between the electrodes and the nanoparticle migration. Therefore, the voltage applied on submicrometre electrodes was increased to $20 \mathrm{~V}$, and the distance between the working and counter electrodes was decreased to $5 \mathrm{~mm}$. The concentration of latex nanoparticles in the solution was $2.5 \mathrm{mg} / \mathrm{ml}$, and experiments were performed with $1 \mathrm{ml}$ of solution.

The directed assembly of nanoparticles of the same type was studied with also silica nanoparticles. The experimental parameters (applied voltage and time) were the same as for latex nanoparticles. However, silica particles did not form aggregations on the electrode surface due to their lower $\zeta$ potential $(\sim-24 \mathrm{mV})$. It can be noticed from Fig. 7 that the particles tend to locate more to the edges of the electrodes with $90 \%$ deposition efficiency. This tendency is the effect of fringing fields on the edges that generates a higher potential on those areas compared to the central parts.

Silica nanoparticles were also successfully patterned on much thinner $(1 \mu \mathrm{m})$ electrodes by utilizing
EBL (shown in Fig. 8). Around $20 \mathrm{~V}$ of potential was applied for $5 \mathrm{~min}$, and the concentration of silica nanoparticles in the solution was $2.5 \mathrm{mg} / \mathrm{ml}$ as in the case of latex nanoparticles. Experiments were carried out by using $1 \mathrm{ml}$ of solution.

Binary patterning of two types of nanoparticles

\section{Deposition and patterning of two types of nanoparticles on different sites on the surface}

The next stage of the study incorporates assembling different types of nanoparticles on adjacent locations at the same surface. The experimental set-up and working mechanism are the same as described earlier. In this study, comb-like electrode structures were used (Fig. 2); in this way, the electrodes next to each other are charged interchangeably. First, $15-\mathrm{V}$ positive potential bias was applied to one side of the electrode with respect to the counter electrode. This leaves every other electrode on the pattern neutral. The electrodes were immersed into
Fig. 7 a, b Fluorescent micrographs of silica nanoparticle patterns on rectangular electrodes
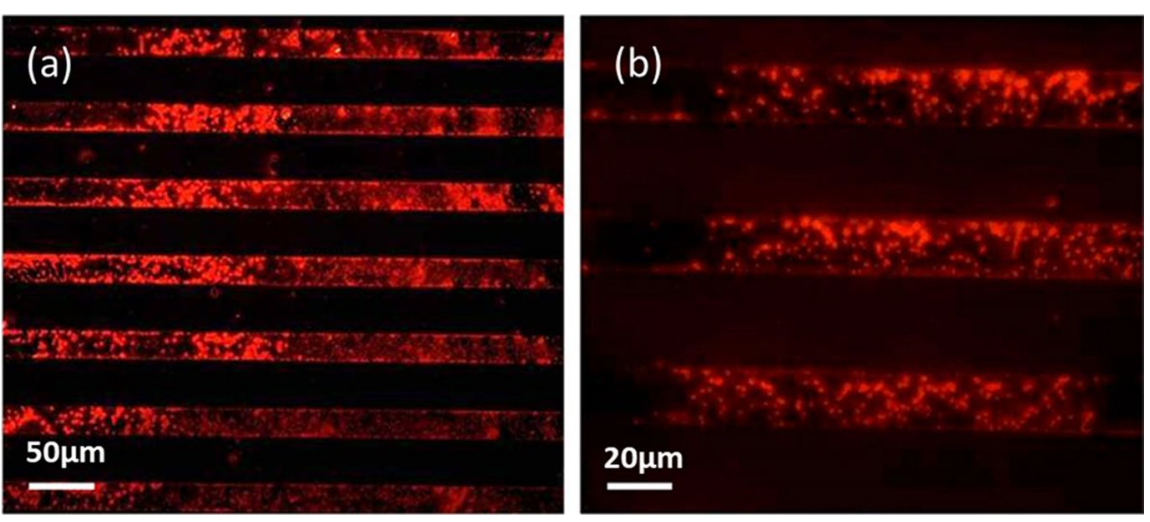

Fig. 8 a, b Silica nanoparticle deposition on $1-\mu \mathrm{m}$ electrode lines
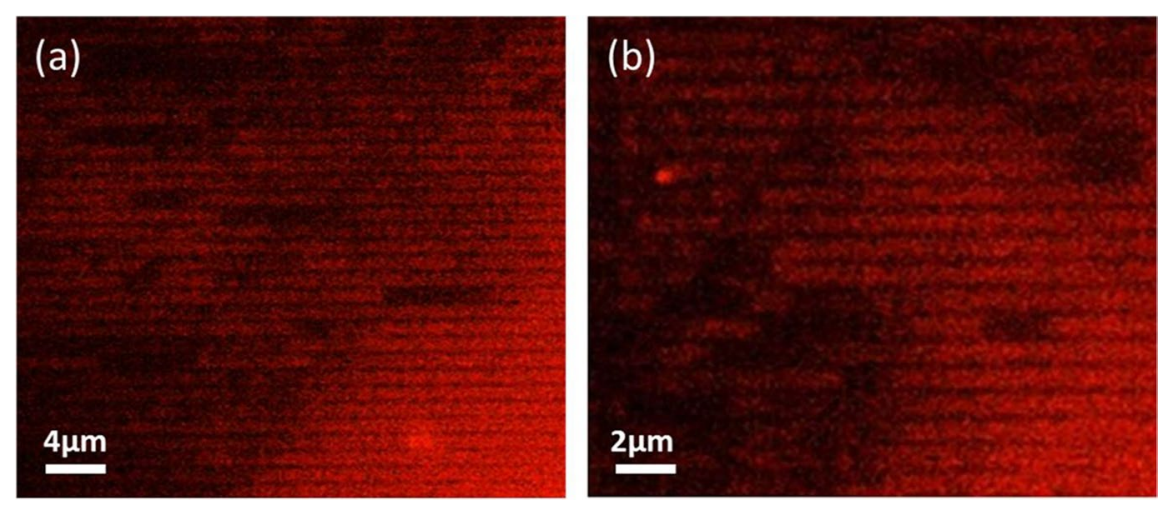
the latex nanoparticle solution for $5 \mathrm{~min}$, and the substrate with patterns was disconnected from the voltage source and was dried under nitrogen flow. Figure 9a confirms that latex nanoparticles were assembled on every other parallel electrode and are positioned only on the areas which were biased with positive potential. After the characterization, the voltage source was connected to the adjacent comb electrode, and positive potential was applied while immersing the electrodes into the silica nanoparticle solution. It should be expected that silica particles should migrate only to the electrode areas which are charged and not get attracted to sites which have latex nanoparticles on them. Nonetheless, it is evident from Fig. 9b that some particles landed on the neighbouring electrodes as well. The density of the particles assembled on the charged surfaces is yet higher. The surfactant of latex particles is a carboxylate group, and they are anionic as it was confirmed with $\zeta$-potential results. On the other hand, the amine capping agent in silica solution is zwitterionic, meaning that it carries both positive and negative surface charges depending on the environment. Therefore, although the net surface potential of silica is negative, it also carries some amount of positive potential. Since latex particles which are already assembled on the electrodes are highly negatively charged, positive charge induced from amine molecules in silica is the cause of attraction towards the latex nanoparticles.
Successive deposition and patterning of two types of nanoparticles on the same sites of the surface

The last stage of this work describes the procedure of obtaining composite surfaces where two different types of nanoparticles are assembled on the same location of the surface. The experimental procedure is similar to previous experiments. The only difference is that instead of using two opposed comb-like electrodes, a single electrode array composed of parallel, connected metal lines were used; hence, the potential was applied to all electrode areas simultaneously. First, electrodes were immersed into the silica nanoparticle solution while $15 \mathrm{~V}$ of positive voltage was applied and then they were immersed into the latex nanoparticle solution for $5 \mathrm{~min}$ without interrupting the voltage bias. After the deposition of latex for $5 \mathrm{~min}$, the sample was dried, and composite structures were obtained successfully. Figure 10a,b confirms that both silica and latex are on the surface of the same electrodes. Silica illuminates red, and latex illuminates a green colour under fluorescent light. The fluorescent microscope that is used for this study cannot display red and green illuminations concurrently; therefore, two images taken from the same area were superimposed to show the composite structure patterns more clearly. From these images, it can be concluded that the electrode surface is coated with a mixture of both silica and latex nanoparticles, and latex is not completely on top of silica as illuminations of
Fig. 9 Assemblies of latex (a) and silica (b) nanoparticles positioned next to each other under a fluorescent microscope; superimposed image (c) of (a) and (b) to demonstrate the two types of particles are on the same surface (our fluorescent microscope cannot display red and green illumination concurrently). (An SEM image showing these two particles deposited on the same location is included in the supplementary document as Figure S6.)
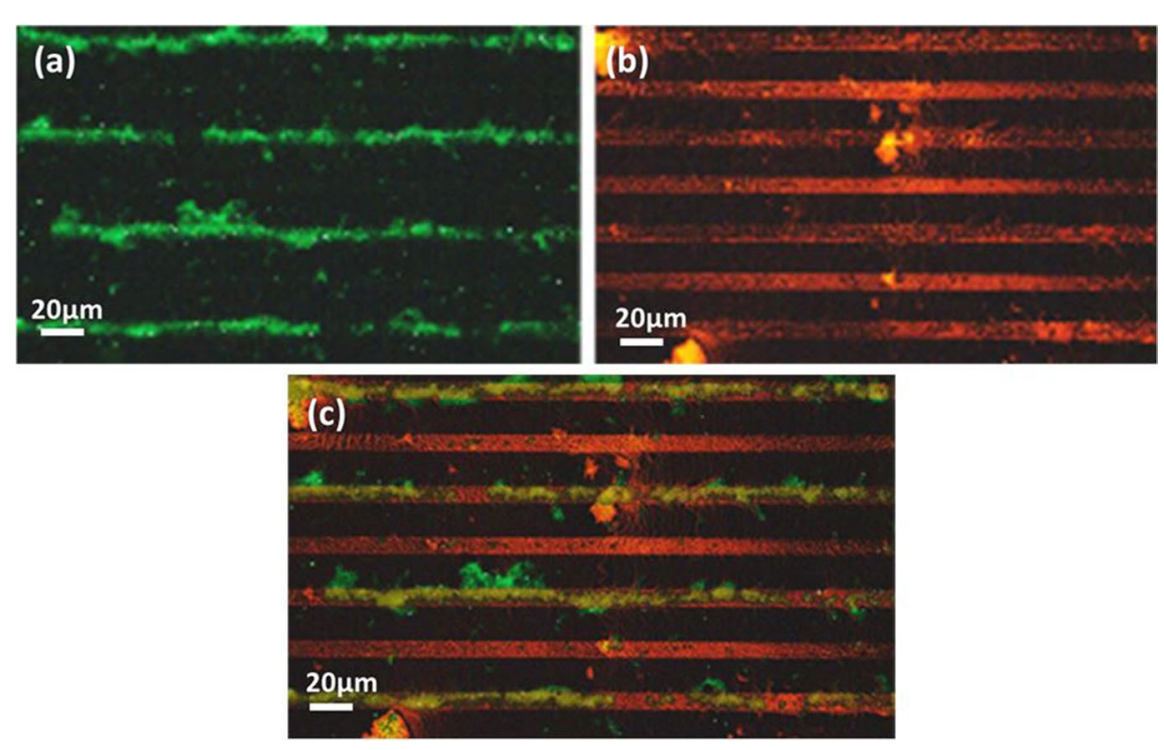
Fig. 10 Composite patterns containing silica (a) and latex nanoparticles (b) assembled on 20 - $\mu$ m wide electrodes; superimposing (c) of (a) and (b) to show that both particles are on the same surface
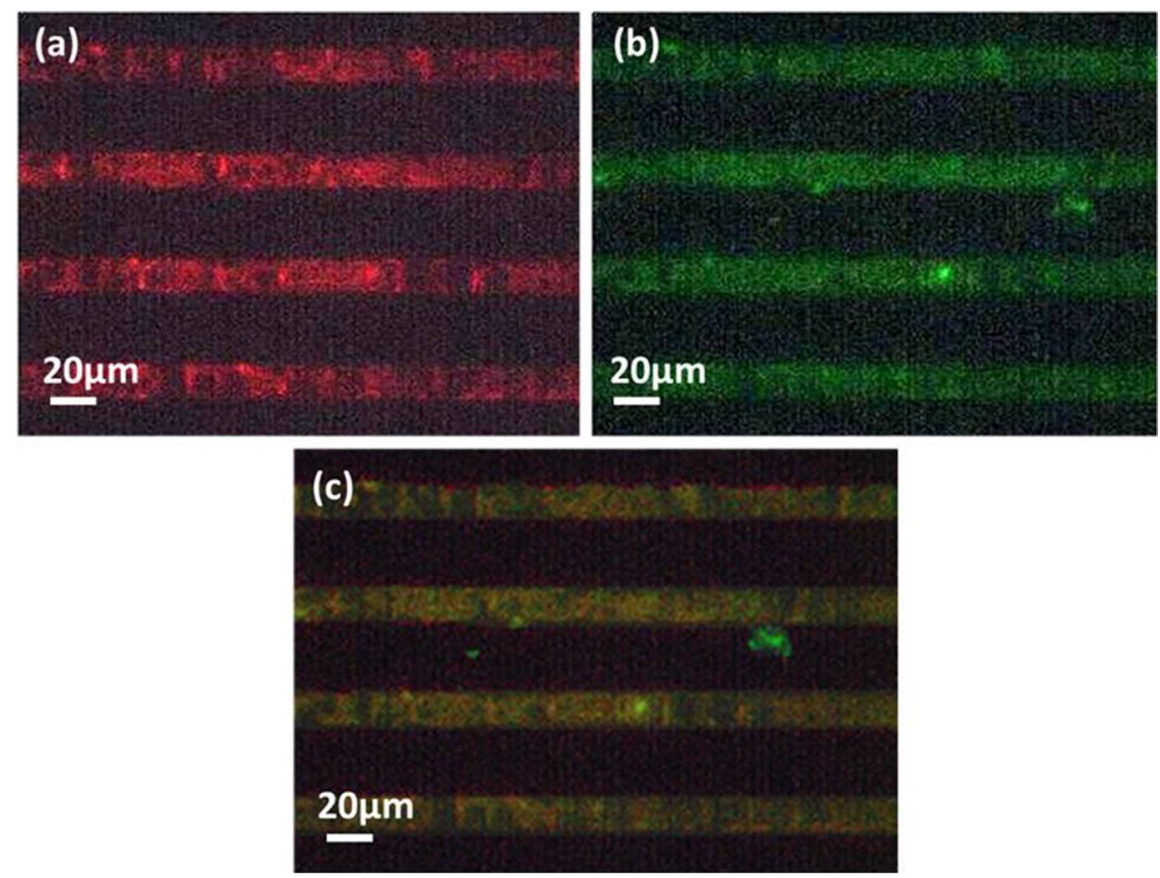

both red and green are observable from the superimposed image.

When the particles are deposited on thinner electrodes with a shorter distance between them, it was observed that latex nanoparticles tend to aggregate, and therefore, the density of the particle areas is not uniform on the surface. However, latex nanoparticles follow the electrode geometry (Fig. 11a). The deposition of silica is more uniform, and the pattern can be observed clearly (Fig. 11b).
Fig. 11 Composite patterns containing a silica and $\mathbf{b}$ latex nanoparticles assembled on 5- $\mu$ m wide electrodes fabricated with EBL; combination (c) of (a) and (b) to show that both particles are on the same surface
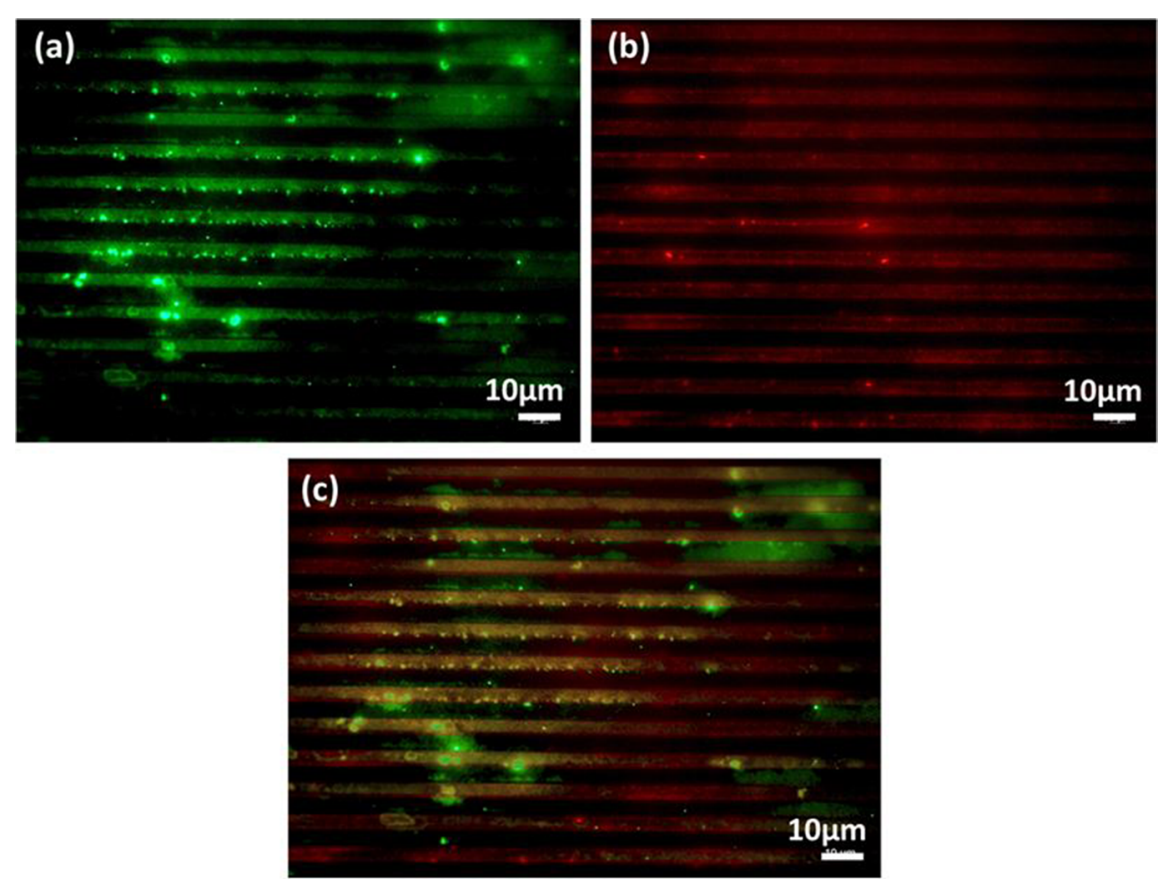
It should be noted that all nanoparticles used in this study were in aqueous solutions, and therefore, pulsed DC voltage (instead of a continuous signal) was applied across the electrodes for all the experiments. Some bubbling due to evaporation was observed on the edges; however, the amount of bubbling was very minimal, and particles mostly adhered on the electrodes rather than the edges due to larger voltage differences. Therefore, the bubbling did not interrupt the nanoparticle diffusion. Nonpolar solvents are favourable to avoid electrolysis when regular DC voltage is applied (as it worked for silver nanoparticles dispersed in ethanol); however, latex and silica nanoparticles became unstable immediately after adding a few droplets of ethanol to the solutions; therefore, the study was continued with aqueous solutions.

\section{Conclusion}

In this work, the patterns of different types of nanoparticles were obtained on surfaces by applying electrostatic forces on charged nanoparticles. At the first stage of the study, assemblies of one type of particles were achieved on the surface. Aqueous dispersions of silver, fluorescent latex and silica nanoparticles were utilized for experimental tests. The results indicate that more precise and finer depositions can be produced on structured metallic surfaces when a large amount of electric field is applied. On the other hand, it was observed that as $\zeta$ potential of the solution is higher, particles got deposited faster. At the second stage, the binary assemblies of latex and silica particles were produced on the desired locations of the surface. First, latex was patterned on one electrode structure followed by patterning silica on the other electrode on the same surface. Due to the comb-like shape of the electrodes, patterns of latex and silica alter on each electrode line. At last, structured composite surfaces were created from silica and latex particles. The patterns of these composited were controlled by the electrode shape.

The method that is used here is applicable for any kind of conductive electrode surface and any kind of charged nanomaterial in a fluidic medium. The patterns of any shape can be produced, and the resolution is dependent on electrode dimensions. This flexibility opens possibilities for designing devices with the desired pattern and nanoparticle composition. For instance, surfaces patterned with different types of nanoparticles can be designed as multiplexed sensing platforms.

Finally, the concentration of nanoparticles used in this study was the same for all experiments; however, it should be noted that this parameter would have an effect on the coverage of printed areas. The effect of this parameter can be studied in future work.

Acknowledgements This project was funded by The Scientific and Technological Council of Turkey under Career Development Program (grant number: 115M571). The authors would like to acknowledge Ms. Çağla Berberoğlu for her assistance with superimposing fluorescent images.

Funding This study was funded by the Scientific and Technological Council of Turkey under Career Development Program (grant number: 115M571).

\section{Declarations}

Conflict of interest The authors declare that they have no conflict of interest.

\section{References}

Abdellatif MH, Salerno M, Abdelrasoul GN, Liakos I, Scarpellini A, Marras S, Diaspro A (2016) Effect of Anderson localization on light emission from gold nanoparticle aggregates. Beilstein J Nanotechnol 7:2013-2022. https:// doi.org/10.3762/bjnano.7.192

An BW, Kim K, Lee H, Kim SY, Shim Y, Lee DY, Song J, Park JU (2015) High-resolution printing of 3D structures using an electrohydrodynamic inkjet with multiple functional inks. Adv Mater 27:4322-4328. https://doi.org/10.1002/ adma.201502092

Banstola A, Emami F, Jeong JH, Yook S (2018) Current applications of gold nanoparticles for medical imaging and as treatment agents for managing pancreatic Cancer. Macromol Res 26:955-964. https://doi.org/10.1007/ s13233-018-6139-4

Barry CR, Steward MG, Lwin NZ, Jacobs HO (2003) Printing nanoparticles from the liquid and gas phases using nanoxerography. Nanotechnology 14:1057-10163. https://doi. org/10.1088/0957-4484/14/10/301

Barry CR, Gu J, Jacobs HO (2005) Charging process and coulomb-force-directed printing of nanoparticles with sub100-nm lateral resolution. Nano Lett 5:2078-2084. https:// doi.org/10.1021/n10511972

Beattie NS, See P, Zoppi G, Ushasree PM, Duchamp M, Farrer I, Ritchie DA, Tomić S (2017) Quantum engineering of InAs/GaAs quantum dot based intermediate band solar cells. ACS Photonics 4:2745-2750. https://doi.org/10. 1021/acsphotonics.7b00673

Belusso LC, Lenz GF, Fiorini EE, Pereira AJ, Sequinel R, Bini RA, Felix JF, Schneider R (2019) Synthesis of 
silver nanoparticles from bottom up approach on borophosphate glass and their applications as SERS, antibacterial and glass-based catalyst. Appl Surf Sci 473:303312. https://doi.org/10.1016/j.apsusc.2018.12.155

Besra L, Uchikoshi T, Suzuki TS, Sakka Y (2009) Application of constant current pulse to suppress bubble incorporation and control deposit morphology during aqueous electrophoretic deposition (EPD). J Eur Ceram Soc 29:1837-1845. https://doi.org/10.1016/j.jeurceramsoc. 2008.07.031

Chavez-Valdez A, Shaffer MS, Boccaccini AR (2013) Applications of graphene electrophoretic deposition. A Review. J Phys Chem B 117:1502-1515. https://doi.org/ 10.1021/jp3064917

Demko MT, Choi S, Zohdi TI, Pisano AP (2011) High resolution patterning of nanoparticles by evaporative self-assembly enabled by in situ creation and mechanical lift-off of a polymer template. Appl Phys Lett 99:253102. https://doi.org/10.1063/1.3671084

Ding F, Fan Y, Sun Y, Zhang F (2019) Beyond 1000 nm emission wavelength: recent advances in organic and inorganic emitters for deep-tissue molecular imaging. Adv Healthcare Mater 8:1900260. https://doi.org/10. 1002/adhm.201900260

Gao M, Sun J, Dulkeith E, Gaponik N, Lemmer U, Feldmann J (2002) Lateral Patterning of CdTe nanocrystal films by the electric field directed layer-by-layer assembly method. Langmuir 18(4098):4102. https://doi.org/10. 1021/1a015599a

Goesmann H, Feldmann C (2010) Nanoparticulate functional materials. Angew Chem Int Ed 49:1362-1395. https:// doi.org/10.1002/anie.200903053

Guo W, Liu B (2012) Liquid-phase pulsed laser ablation and electrophoretic deposition for chalcopyrite thinfilm solar cell application. ACS Appl Mater Interfaces 4:7036-7042. https://doi.org/10.1021/am3022976

Hamaker H (1940) Formation of a deposit by electrophoresis. Trans Faraday Soc 35:279-287. https://doi.org/10. 1039/TF9403500279

Hunter AC, Elsom J, Wibroe PP, Moghimi SM (2012) Polymeric particulate technologies for oral drug delivery and targeting: a pathophysiological perspective. Maturitas 73:5-18. https://doi.org/10.1016/j.maturitas.2012.05. 014

Intartaglia R, Rodio M, Abdellatif M, Prato M, Salerno M (2016) Extensive characterization of oxide-coated colloidal gold nanoparticles synthesized by laser ablation in liquid. Materials 9:775. https://doi.org/10.3390/ma9090775

Jacobs HO, Campbell SA, Steward MG (2002) Approaching nanoxerography: the use of electrostatic forces to position nanoparticles with $100 \mathrm{~nm}$ scale resolution. Adv Mater 14:1553-1557. https://doi.org/10.1002/1521-4095(20021 104)14:21\%3c1553::AID-ADMA1553\%3e3.0.CO;2-9

Jeong JW, Park WI, Do LM, Park JH, Kim TH, Chae G, Jung YS (2012) Nanotransfer printing with sub-10 nm resolution realized using directed self-assembly. Adv Mater 24:3526-3531. https://doi.org/10.1002/adma.201200356

Jiang X, Feng J, Huang L, Wu Y, Su B, Yang W, Mai L, Jiang L (2016) Bioinspired 1D superparamagnetic magnetite arrays with magnetic field perception. Adv Mater 28:6952-6958. https://doi.org/10.1002/adma.201601609
Kamyshny A, Magdassi S (2014) Conductive nanomaterials for printed electronics. Small 10:3515-3535. https://doi.org/ 10.1002/smll.201303000

Liu Z, Lin CH, Hyun BR, Sher CW, Lv Z, Luo B, Jiang F, Wu T, Ho CH, Kuo HC, He JH (2020) Micro-light-emitting diodes with quantum dots in display technology. Light Sci Appl 9:83. https://doi.org/10.1038/s41377-020-0268-1

Mills SC, Smith CS, Arnold DP, Andrew JS (2020) Electrophoretic deposition of iron oxidenanoparticles to achieve thick nickel/iron oxidemagnetic nanocomposite films. AIP Adv 10:015308. https://doi.org/10.1063/1.5129797

Morales D, Teulon L, Palleau E, Alnasser T, Ressier L (2018) Single-step binary electrostatic directed assembly of active nanogels for smart concentration-dependent encryption. Langmuir 34:1557-1563. https://doi.org/10. 1021/acs.langmuir.7b03519

Moutet P, Lacroix LM, Robert A, Impéror-Clerc M, Viau G, Ressier L (2015) Directed assembly of single colloidal gold nanowiresby AFM nanoxerography. Langmuir 31:4106-4112. https://doi.org/10.1021/acs.langmuir.5b002 99

Murray CB, Norris DJ, Bawendi MG (1993) Synthesis and characterization of nearly monodisperse $\mathrm{CdE}(\mathrm{E}=$ sulfur, selenium, tellurium) semiconductor nanocrystallites. JACS 115:8706-8715. https://doi.org/10.1021/ja000 $72 \mathrm{a} 025$

Oberdick SD, Majetich SA (2013) Electrophoretic deposition of iron oxide nanoparticles on templates. J Phys Chem C 117:18709-18718. https://doi.org/10.1021/jp405395y

Palleau E, Ressier L, Mélin T (2010) Numerical simulations for a quantitative analysis of AFM electrostatic nanopatterning on PMMA by Kelvin force microscopy. Nanotechnology 21:225706. https://doi.org/10.1088/0957-4484/21/ 22/225706

Palleau E, Sangeetha NM, Viau G, Marty JD, Ressier L (2011) Coulomb force directed single and binary assembly of nanoparticles from aqueous dispersions by AFM nanoxerography. ACS Nano 5:4228-4235. https://doi.org/10. 1021/nn2011893

Pankhurst Q, Jones S, Dobson J (2016) Applications of magnetic nanoparticles in biomedicine: the story so far. J Phys D: Appl Phys 49:501002

Park I, Ko SH, Pan H, Grigoropoulos CP, Pisano AP, Fréchet JMJ, Lee ES, Jeong JH (2008) Nanoscale patterning and electronics on flexible substrate by direct nanoimprinting of metallic nanoparticles. Adv Mater 20:489-496. https:// doi.org/10.1002/adma.200702326

Park W, Heo YJ, Han DK (2018) New opportunities for nanoparticles in cancer immunotherapy. Biomater Res 22:24. https://doi.org/10.1186/s40824-018-0133-y

Peng J, Li J, Xu W, Wang L, Su D, Teoh CL, Chang YT (2018) Silica nanoparticle-enhanced fluorescent sensor array for heavy metal ions detection in colloid solution. Anal Chem 90:1628-1634. https://doi.org/10.1021/acs.analchem.7b028 83

Qian F, Pascall AJ, Bora M, Han TYJ, Guo S, Ly SS, Worsley MA, Kuntz JD, Olson TY (2015) On-demand and location selective particle assembly via electrophoretic deposition for fabricating structures with particle-to-particle precision. Langmuir 31:3563-3568. https://doi.org/10.1021/la502724n 
Sarkar P, Nicholson PS (1996) Electrophoretic deposition (EPD): mechanisms, kinetics, and application to ceramics. JACS 79:1987-2002. https://doi.org/10.1111/j.1151-2916. 1996.tb08929.x

Selopal GS, Zhao H, Tong X, Benetti D, Navarro-Pardo F, Zhou Y, Barba D, Vidal F, Wang ZM, Rosei F (2017) Highly stable colloidal "giant" quantum dots sensitized solar cells. Adv Funct Mater 27:1701468. https://doi.org/ 10.1002/adfm.201701468

Seuss S, Boccaccini AR (2013) Electrophoretic deposition of biological macromolecules, drugs, and cells. Biomacromol 14:3355-3369. https://doi.org/10.1021/bm401021b

Sharonova A, Loza K, Surmeneva M, Surmenev R, Prymak O, Epple M (2016) Synthesis of positively and negatively charged silver nanoparticles and their deposition on the surface of titanium. IOP Conf Ser: Mater Sci Eng 116:012009. https://doi.org/10.1088/1757-899X/116/1/012009

Shen K, Li X, Xu H, Wang M, Dai X, Guo J, Zhang T, Li S, Zou G, Choy KL, Parkin IP, Guo Z, Liu H, Wu J (2019) Enhanced performance of $\mathrm{ZnO}$ nanoparticle decorated allinorganic $\mathrm{Cs} \mathrm{PbBr} 3$ quantum dot photodetectors. J Mater Chem A 7:6134-6142. https://doi.org/10.1039/C9TA0 $0230 \mathrm{H}$

Sikkema R, Baker K, Zhitomirsky I (2020) Electrophoretic deposition of polymers and proteins for biomedical applications Adv. Colloid Interface Sci 284:102272. https://doi. org/10.1016/j.cis.2020.102272
Song W, Li H, Liang H, Qiang W, Xu D (2014) Disposable electrochemical aptasensor array by using in situ DNA hybridization inducing silver nanoparticles aggregate for signal amplification. Anal Chem 86:2775-2783. https:// doi.org/10.1021/ac500011k

Terris BD, Thomson T (2005) Nanofabricated and self-assembled magnetic structures as data storage media. J Phys D: Appl Phys 38:R199

Wood NR, Wolsiefer AI, Cohn RW, Williams SJ (2013) Dielectrophoretic trapping of nanoparticles with an electrokinetic nanoprobe. Electrophoresis 34:1922-1930. https:// doi.org/10.1002/elps.201300004

Yoo J, Han S, Park W, Lee T, Park Ym Chang H, Hahn SK, Kwon W (2018) ACS Appl. Mater Interfaces 10:4424744256. https://doi.org/10.1021/acsami.8b16163

Zhang H, Cadusc J, Kinnear C, James T, Roberts A, Mulcaney P (2018a) Direct assembly of large area nanoparticle arrays. ACS Nano 12:7529-7537. https://doi.org/10.1021/ acsnano.8b02932

Zhang H, Cadusch J, Kinnear C, James T, Roberts A, Mulvaney P (2018b) Direct assembly of large area nanoparticle arrays. ACS Nano 12:75297537

Publisher's note Springer Nature remains neutral with regard to jurisdictional claims in published maps and institutional affiliations. 